\title{
The Functional Crosstalk between MT1-MMP and Adams
}

\section{Zhongjun Zhou* and Hoi Leong Xavier Wong}

Department of Biochemistry, LKS Faculty of Medicine, University of Hong Kong

Matrix Metalloproteinases (MMPs) are essential for proper physiological tissue remodeling and homeostasis. They have a broad spectrum of substrates, ranging from extracellular matrix proteins to cytokines and membrane bound proteins [1]. One of the MMPs that draw worldwide research interests is membrane-type-1 metalloproteinase (MT1-MMP; also known as matrix metalloproteinase 14, MMP14). The physiological significance of MT1-MMP can be revealed by the severe phenotypes identified in MT1-MMP deficient mice that exhibit dwarfism, retarded skeletal growth, impaired branching morphogenesis in lung and submandibular gland, defective vascularization in endochondral ossification and cornea, and compromised adipogenesis in white adipose tissues [2-5]. Most previous studies on MT1-MMP have suggested that these developmental defects are attributable to impaired remodeling of type-I collagen [3]. However, a distinct mechanism by which MT1-MMP regulates intramembranous ossification is recently reported. The functional role of MT1-MMP in calvarial development is no longer to be restricted to extracellular remodeling, but also is applicable to the modulation of cellular signaling events essential for Calvarial Osteoblast (CO) development. We recently demonstrated a cell-autonomous and positive regulatory role of MT1-MMP in calvarial development via the modulation of Fibroblast Growth Factor (FGF) signaling pathway [6].

FGF signaling plays a fundamental role in craniofacial development. It regulates a variety of cellular events, particular in $\mathrm{CO}$ proliferation, differentiation and cell fate determination during calvarial bone formation [7]. Inactivation of FGF signaling resulted from genetic knockout of FGFRs or FGF ligands leads to dwarfism with defective ossification that can also be observed in Mmp14-/- mice [8-10]. The high degree of similarity in craniofacial defects between Mmp14-/mice and mice with defective FGF signaling highlights the possible functional crosstalk between MT1-MMP and FGF signaling cascade. Indeed, examination of Mmp14-/- mice revealed that defective FGFR2mediated signaling in $\mathrm{CO}$ development is the predominant mechanism leading to defects in intramembranous ossification of Mmp14-/- mice during embryonic development, implying that MT1-MMP is a potent positive modulator of FGF signaling.

MT1-MMP does not act alone on FGF signaling but rather functionally work with another metalloproteinase family, ADAMs. MT1-MMP proteolytically inactivates ADAM9 to protect FGFR2 from ectodomain shedding of ADAM9, which is important for the maintenance of optimal FGF signaling during calvarial development. The importance of ADAM9 in MT1-MMP/FGFR2 signaling cascade is further strengthened by the efficient rescue of calvarial defects of Mmp14-/- mice via depletion of ADAM9. The discovery of involvement of ADAM9 in MT1-MMP regulatory loop for FGF signaling not only provides a new molecular insight into the mechanism by which FGF signaling is regulated during intramembranous ossification, but also establishes a new research interest in which the newly identified crosstalk between MMP and ADAM families will be of great importance.

ADAM9 is not the solo ADAM family member regulated by MT1MMP. We recently reported that ADAM15 is also proteolytically cleaved by MT1-MMP [11]. However, how the regulation of ADAM15 by MT1MMP contributes to physiological development or pathologies remains largely unknown. It is clear that ADAM15 has a broad spectrum of substrates involved in many important physiological and pathological events, such as neovascularization, bone development, inflammation, cancer progression and metastasis [12-14]. It will be evitable to ask whether the interplay between ADAM15 and MT1-MMP plays a role in any physiological processes in which MT1-MMP is indispensable.

The newly-identified mechanism by which MT1-MMP regulates $\mathrm{CO}$ development suggests new therapeutic possibilities. Manipulation of MT1-MMP/ADAM9/FGFR2 signaling pathway will provide new approaches for the treatment of skeletal disorders and many cancers associated with deregulated FGF signaling.

\section{References}

1. Page-McCaw A, Ewald AJ, Werb Z (2007) Matrix metalloproteinases and the regulation of tissue remodelling. Nat Rev Mol Cell Biol 8: 221-233.

2. Oblander SA, Zhou Z, Galvez BG, Starcher B, Shannon JM, et al. (2005) Distinctive functions of membrane type 1 matrix-metalloprotease (MT1-MMP or MMP-14) in lung and submandibular gland development are independent of its role in pro-MMP-2 activation. Dev Biol 277: 255-269.

3. Holmbeck K, Bianco P, Caterina J, Yamada S, Kromer M, et al. (1999) MT1MMP-deficient mice develop dwarfism, osteopenia, arthritis, and connective tissue disease due to inadequate collagen turnover. Cell 99: 81-92.

4. Zhou Z, Apte SS, Soininen R, Cao R, Baaklini GY, et al. (2000) Impaired endochondral ossification and angiogenesis in mice deficient in membranetype matrix metalloproteinase I. Proc Natl Acad Sci U S A 97: 4052-4057.

5. Chun TH, Hotary KB, Sabeh F, Saltiel AR, Allen ED, et al. (2006) A pericellula collagenase directs the 3-dimensional development of white adipose tissue. Cell 125: 577-591.

6. Chan KM, Wong HL, Jin G, Liu B, Cao R, et al. (2012) MT1-MMP Inactivates ADAM9 to Regulate FGFR2 Signaling and Calvarial Osteogenesis. Dev Cell.

7. Ornitz DM, Marie PJ (2002) FGF signalling pathways in endochondral and intramembranous bone development and human genetic disease. Genes Dev 16: $1446-1465$

8. Liu Z, Lavine KJ, Hung IH, Ornitz DM (2007) FGF18 is required for early chondrocyte proliferation, hypertrophy and vascular invasion of the growth plate. Dev Biol 302:80-91.

9. Ohbayashi N, Shibayama M, Kurotaki Y, Imanishi M, Fujimori T, et al (2002) FGF18 is required for normal cell proliferation and differentiation during osteogenesis and chondrogenesis. Genes Dev 16: 870-879.

10. Montero A, Okada Y, Tomita M, Ito M, Tsurukami H, et al. (2000) Disruption of the fibroblast growth factor-2 gene results in decreased bone mass and bone formation. J Clin Invest 105: 1085-1093.

11. Wong HL, Cao R, Jin G, Chan KM, Cao Y et al. (2012) When MT1-MMP meets ADAMs. Cell Cycle 11.

*Corresponding author: Zhongjun Zhou, Department of Biochemistry, LKS Faculty of Medicine, University of Hong Kong, E-mail: zhongjun@hkucc.hku.hk

Received August 22, 2012; Accepted August 22, 2012; Published August 24 2012

Citation: Zhou Z, Wong HLX (2012) The Functional Crosstalk between MT1-MMP and Adams. Biochem Anal Biochem 1:e113. doi:10.4172/2161-1009.1000e113

Copyright: () 2012 Zhou Z, et al. This is an open-access article distributed under the terms of the Creative Commons Attribution License, which permits unrestricted use, distribution, and reproduction in any medium, provided the original author and source are credited. 
Citation: Zhou Z, Wong HLX (2012) The Functional Crosstalk between MT1-MMP and Adams. Biochem Anal Biochem 1:e113. doi:10.4172/21611009.1000e113

Page 2 of 2

12. Lucas N, Najy AJ, Day ML (2009) The therapeutic potential of ADAM15. Curr Pharm Des 15: 2311-2318.

13. Marzia M, Guaiquil V, Horne WC, Blobel CP, Baron R, et al. Lack of ADAM15 in mice is associated with increased osteoblast function and bone mass. Biol Chem 392: 877-885

14. Lucas N, Day ML (2009) The role of the disintegrin metalloproteinase ADAM15 in prostate cancer progression. J Cell Biochem 106:967-974. 